$$
\text { B. Beispiel für }++ \text {-Reaktion }
$$

$1 \mathrm{~cm}^{3}$ AS thermophilum $35^{\circ}$ 13-mal versetzt mit insgesamt $2,4 \mathrm{mg} \mathrm{N}$ AG vulgare

$\begin{array}{ccc}\text { Hälfte } & \text { Getestet mit } 0,1 \mathrm{mg} \mathrm{N} \mathrm{AG} & \begin{array}{c}\text { Präzipitat N } \\ {[\mathrm{mg}]}\end{array} \\ \text { 1. } & \text { vulgare } & \text { keins } \\ 2 . & \text { thermophilum } 35^{\circ} & 0,082\end{array}$

C. Beispiel für +++ -Reaktion

$1,5 \mathrm{~cm}^{3}$ AS dahlemense 18-mal versetzt mit insgesamt $3,35 \mathrm{mg} \mathrm{N} \mathrm{AG}$ vulgare
Drittel Getestet mit 0,1 mg N AG

Präzipitat N

[mg]

1. vulgare keins

2. dahlemense 0,150

3. revirescens keins

Fräulein Lieselotte Siska danke ich für die zuverlässige Unterstützung bei der Durchführung der vorstehenden Untersuchungen, Herrn Professor Dr. H. Friedrich-Freksa und Herrn Dr. E. Weiler für einführende Beratung bei der Anwendung der serologischen Methoden, Herrn Professor Dr. G. Melchers für die Diskussion der Befunde.

\title{
Glutaminsäure in Chlorella
}

\author{
Von Otto Warburg, Helmut Klotzsch und Günter Krippahl \\ Aus dem Max-Planck-Institut für Zellphysiologie, Berlin-Dahlem \\ (Z. Naturforschg. 12 b, 622 -628 [1957] ; eingegangen am 10. August 1957)
}

\begin{abstract}
Versuche über Zerfall und Wiederaufbau der Glutaminsäure in lebender Chlorella und über den Zusammenhang zwischen Glutaminsäure und Photosynthese.
\end{abstract}

Wie bereits kurz mitgeteilt ${ }^{1}$, stammt die Kohlensäure, die Fluorid aus lebender Chlorella entwickelt ${ }^{2}$, aus der $\alpha$-Decarboxylierung der Glutaminsäure:

Glutaminsäure $=\gamma$-Aminobuttersäure $+\mathrm{CO}_{2}$ eine Reaktion, die Ackermann ${ }^{3} 1910$ in Bakterien entdeckte und die seitdem weitverbreitet in Tierund Pflanzenreich gefunden worden ist ${ }^{4-7}$.

Auch gefroren-getrocknete Chlorella ist als Ferment dieser Reaktion wirksam, jedoch wesentlich schwächer, als lebende Chlorella. Bei $20^{\circ}$ ist die Halbwertzeit für $100 \mathrm{~mm}^{3}$ lebende Chlorella $5 \mathrm{Min}$., für die daraus gewonnene Trockensubstanz 50 Minuten.

$\mathrm{Da}$ die erhebliche und schnelle Kohlensäure-Entwicklung, die bei Zusatz von Fluorid zu lebender Chlorella gefunden wird, von einer Decarboxylierung der Glutaminsäure herrührt, ist durch Manometrie gefunden und durch Papierchromatographie bestätigt worden. Immer, wenn Fluorid Kohlensäure aus Chlorella entwickelt, nimmt die Glutaminsäure $\mathrm{ab}$ und die $\gamma$-Aminobuttersäure nimmt $\mathrm{zu}$; und immer, wenn die Fluoridwirkung gehemmt wird,

1 O. Warburg, H. Klotzsch u. G. Krippahl, Z. Naturforschg. 12 b, 266 [1957].

2 O. Warburg u. G. Krippahl, Z. Naturforschg. 11 b, 718 [1956].

3 D. Ackermann, Hoppe-Seyler's Z. physiol. Chem. 69, 279 [1910]. z. B. durch Blausäure oder durch Sauerstoff, wird auch die Zersetzung der Glutaminsäure gehemmt.

Chlorella enthält je nach der Züchtung 0,5 bis 1,3\% ihrer Trockensubstanz an Glutaminsäure, und zwar um so mehr Glutaminsäure, als sie Chlorophyll enthält. Beträgt der Chlorophyllgehalt 5 bis $8 \%$, so kommt 1 Mol. bis 2 Mole. Glutaminsäure auf 1 Mol. Chlorophyll. Wahrscheinlich ist in diesen Zellen alle Glutaminsäure an Chlorophyll gebunden. Sehr chlorophyllarme Zellen enthalten mehr Glutaminsäure, als Chlorophyll. Wahrscheinlich ist in diesen Zellen ein Teil der Glutaminsäure frei, ein Teil an Chlorophyll gebunden.

Es ist eine sehr wichtige Tatsache, daß Sauerstoff die Zersetzung der Glutaminsäure durch Fluorid hemmt. Die Erklärung ist, daß bei Zusatz von Fluorid die Atmung steigt und daß durch die Energie der gesteigerten Atmung die zersetzte Glutaminsäure wieder soweit aufgebaut wird, bis Zersetzung durch Fluorid und Wiederaufbau durch die Atmung sich die Waage halten. Zum Beispiel zersetzt $n / 1000$ Fluorid anaerob die Glutaminsäure vollständig,

${ }^{4}$ К. Окоnикi, Botan. Mag. [Tokio] 51, 270 [1937].

5 E. S. TAYlor u. E. F. Gale, Bioch. J. 39, 52 [1945].

6 O. Schales, V. Mims u. S. Schales, Arch. Biochemistry 10, $455 ; 11,155$ [1946].

7 E. Roberts u. S. Frankel, J. biol. Chemistry 190, 505 [1951]. 
aerob aber, wegen des Wiederaufbaus, nur zu einem kleinen Teil.

Zersetzung und Wiederaufbau der Glutaminsäure in lebender Chlorella, die durch Fluoridzusatz manifest werden, sind Reaktionen die einen Umsatz von der Größenordnung des Energie-Stoffwechsels haben. Die Idee, daß sie im Mechanismus der Photosynthese eine Rolle spielen, wird nahegelegt durch die Analogie der durch Licht und durch Fluorid induzierten Atmung, deren Energie in beiden Fällen zu Reaktionen benutzt wird, an denen die Kohlensäure beteiligt ist.

Man kann diese Idee prüfen, indem man die Glutaminsäure zersetzt und dann die Lichtwirkung mißt. Es zeigt sich dann, daß die Lichtwirkung in dem Maß sinkt, als die Glutaminsäure zersetzt wird; und daß die Lichtwirkung in dem Maße wieder steigt, in dem die Glutaminsäure wieder aufgebaut wird.

Die Beteiligung der Glutaminsäure an der Wirkung des Lichts scheint uns durch derartige Versuche bewiesen zu sein, doch ist auf Grund von Versuchen ${ }^{8}$ mit radioaktiver Kohlensäure daran zu denken, daß ein System Asparaginsäure-Alanin der Glutaminsäure vorgeschaltet ist, da bei Belichtung die Radioaktivität früher in Asparaginsäure als in Glutaminsäure erscheint.

Asparagin kommt in sehr kleinen Mengen, Alanin in größeren Mengen in Chlorella vor. Eine Asparaginsäure-Decarboxylase haben wir bisher in Chlorella nicht nachweisen können.

Die beiden letzten Abschnitte dieser Arbeit betreffen die dissoziierende Kohlensäure der Chlorella, insbesondere diejenige dissoziierende Kohlensäure, die nur bei Gegenwart von Sauerstoff gefunden wird und die Beziehungen zur Glutaminsäure zu haben scheint. Während wir bei Belichtung keine Abnahme der Glutaminsäure in Chlorella gefunden haben, zeigte es sich, daß die dissoziierende Kohlensäure, wenn im Licht Sauerstoff entwickelt wird, erheblich abnimmt und in stark belichteten Zellen völlig verschwindet. Verdunkelt man dann wieder, so steigt die aerobe dissoziierende Kohlensäure im Verlauf von 20 Min. - das ist gerade die Zeit, in der die lichtinduzierte Atmung abklingt ${ }^{9}$ - wieder auf ihren Dunkelwert.

8 O. Warburg, H. Klotzsch u. G. Krippahl, Z. Naturforschg. 12 b, 481 [1957].

9 O. Warburg, G. Krippahl, W. Schröder u. W. Buchholz, Z. Naturforschg. 9 b, 769 [1954]; O. W ARburg u. W. Schröder, Z. Naturforschg. 10 b, 639 [1955].

\section{Die Versuchszellen und Versuchsmedien}

Chlorella wurde in dem früher angegebenen Salzmedium ${ }^{10}$, unter Zusatz der Mikroelemente, in $250-\mathrm{mm}^{3}$. Kolben, unter Durchleitung von 5 Vol.-Proz. $\mathrm{CO}_{2}$ 30 Vol.-Proz. $\mathrm{O}_{2} 65$ Vol.-Proz. Argon, bei $25^{\circ}$ gezüchtet. Belichtet wurde mit Tageslicht + Metallfadenlicht, oder nur mit Metallfadenlicht, kontinuierlich oder fluktuierend $^{11}$. Der Chlorophyllgehalt der Zellen hängt von der Lichtintensität bei der Zucht ab und wurde von $1 \%$ bis $8 \%$ der Trockensubstanz variiert. Wir bezeichnen in dieser Arbeit die verschiedenen Kulturen folgendermaßen:

$\mathrm{S} \ddot{\mathrm{u}} \mathrm{z}$ z e l l e n. Sie wurden an einem Südfenster unter Zusatz von $300 \mathrm{~W}$ Metallfadenlicht am Tage und $200 \mathrm{~W}$ Metallfadenlicht nachts gezüchtet. Sie vermehrten sich in $24 \mathrm{Stdn}$. von 60 auf $350 \mathrm{~mm}^{3}$ und in $48 \mathrm{Stdn}$. auf $650 \mathrm{~mm}^{3}$. Die 2-tägigen Südzellen enthalten 7 bis $8 \%$ ihres Trockengewichtes an Chlorophyll. Auf $1 \mathrm{Mol}$. Chlorophyll kommt in diesen Zellen fast genau 1 Mol. Glutaminsäure.

Nordzellen. Sie wurden ohne Zusatz von Tageslicht, in einem gegen außen verdunkelten Raum, mit $2 \cdot 200 \mathrm{~W}$ Metallfadenlicht gezüchtet und vermehrten sich in $48 \mathrm{Stdn}$. von 60 auf $1500 \mathrm{~mm}^{3}$. Sie enthalten 5 bis 6\% ihres Trockengewichts an Chlorophyll. Auf $1 \mathrm{Mol}$. Chlorophyll kommt in diesen Zellen 1 Mol. bis 2 Mol. Glutaminsäure.

A - Z e 11 e n. Sie wurden ohne Tageslicht mit fluktuierendem Metallfadenlicht (300-W-Lampe, Spannung $3 \mathrm{Stdn}$. ansteigend von 50 auf $220 \mathrm{~V}$, 9 Stdn. $220 \mathrm{~V}$, $3 \mathrm{Stdn}$. absteigend, $9 \mathrm{Stdn}$. $50 \mathrm{~V})$ gezüchtet und vermehrten sich in $24 \mathrm{Stdn}$. von 60 auf $160 \mathrm{~mm}^{3}$, in $48 \mathrm{Stdn}$. auf $350 \mathrm{~mm}^{3}$. Die 1-tägigen A-Zellen haben $4 \%$ ihres Trockengewichts an Chlorophyll, die 2-tägigen haben $6 \%$ Chlorophyll. In diesen Zellen kommen etwa 1,5 Mole Glutaminsäure auf $1 \mathrm{Mol}$ Chlorophyll.

x-Zellen. Sie entstehen, wenn die Kulturen sehr stark, ohne Tageslicht, mit $4 \cdot 200$-W-Metallfadenlampen belichtet werden, die von zwei Seiten nahe an die Kulturkolben herangebracht werden. Sie vermehren sich in $24 \mathrm{Stdn}$. von 60 auf $200 \mathrm{~mm}^{3}$. Sie enthalten etwa 1,2\% Chlorophyll und 3- bis 5-mal soviel Mole Glutaminsäure als Chlorophyll.

Die $x$-Zellen verhalten sich auch sonst atypisch. Bringt man sie unter anaerobe Bedingungen, so bilden sie viel mehr und viel schneller Milchsäure als die andern Zellen. Die Folge davon ist, daß wegen der Säuerung anaerob bald eine Decarboxylierung der Glutaminsäure einsetzt, deren Kohlensäure von früheren Autoren irrtümlicherweise auf alkoholische Gärung zurückgeführt worden ist. Tatsächlich ist die Gärung der Chlorella eine reine Milchsäuregärung. Merkwürdigerweise aber ist diese Milchsäure nicht L-, sondern D-Milch-

10 Vorschrift s. Z. Naturforschg. 10 b, 631 [1955].

11 O. Warburg, W. Schröder u. H. W. Gattung, Z. Naturforschg. 11 b, 654 [1956]. 
säure, wie in einer Arbeit mit Gewitz und Voelker gezeigt werden wird. Was die Wirkung des Lichts in diesen Zellen anbetrifft, so ist sie mindestens 10-mal geringer als in den andern Zellen, offenbar als Folge einer Gegenreaktion der Zellen gegen die Überbelichtung bei der Zucht. - Die x-Zellen sind als Versuchsmaterial nützlich, wenn man die Glutaminsäure in Chlorella ohne $\mathrm{Zu}$ satz von Fluorid zersetzen will. Wegen der Milchsäurebildung wird in diesen Zellen anaerob, ohne Fluorid, Glutaminsäure zersetzt, aerob wird die Glutaminsäure wieder aufgebaut.

Versuchsmedien. Für die Versuche wurden die Zellen aus der Kulturlösung in einfachere Medien übertragen, in die Salzlösungen $S$ oder $\mathrm{S}^{*}$.

$\mathrm{S}$ a lzlösung S. $10 \mathrm{~g} \mathrm{MgSO}_{4} \cdot 7 \mathrm{H}_{2} \mathrm{O}+5 \mathrm{~g} \mathrm{KH}_{2} \mathrm{PO}_{4}$ $+4 \mathrm{~g} \mathrm{NaCl}+0,4 \mathrm{~g} \mathrm{NH}{ }_{4} \mathrm{Cl}+2 l$ Wasser $+0,6 \mathrm{~cm}^{3}$ $n-\mathrm{H}_{2} \mathrm{SO}_{4} \cdot p_{\mathrm{H}} 3,8$.

Salzlösung S*. $10 \mathrm{~g} \mathrm{MgSO}_{4} \cdot 7 \mathrm{H}_{2} \mathrm{O}+5 \mathrm{~g} \mathrm{KH}_{2} \mathrm{PO}_{4}$ $+2 l$ Wasser $+0,6 \mathrm{~cm}^{3} n-\mathrm{H}_{2} \mathrm{SO}_{4} \cdot p_{\mathrm{H}} 3,8$.

$\mathrm{S}$ a lzlö su ng e n $1 / 10 \mathrm{~S}$ oder $1 / 10 \mathrm{~S}^{*}$. Dies waren die Salzlösungen $S$ oder $\mathrm{S}^{*}$, mit Wasser auf das 10 fache verdünnt. 1/10 $\mathrm{S}^{*}$ wurde vielfach für papierchromatographische Versuche verwendet, bei denen ein höherer Salzgehalt stört. Dest. Wasser schädigt Chlorella und darf als Suspensionsmittel bei biologischen Reaktionen nicht verwendet werden. Wurden die stärkeren Salzlösungen $S$ und $S^{*}$ bei papierchromatischen Versuchen verwendet, so wurde nach Ablauf der biologischen Reaktion zentrifugiert, die Salzlösung durch Wasser ersetzt, und $10 \mathrm{Min}$. auf $90^{\circ}$ erhitzt und die überstehende Lösung eingetrocknet.

\section{Glutaminsäure, manometrisch mit Zell- Trockenpulver}

Chlorella, suspendiert in dest. Wasser, wurde in gefrorenem Zustand getrocknet. In dem Trockenpulver sind die Formen der Zellen erhalten, jedoch sind die Zellen nicht mehr teilungsfähig und nicht mehr imstande, im Licht Kohlensäure zu reduzieren. Das Trockenpulver, suspendiert in Wasser oder Salzlösungen, decarboxyliert L-Glutaminsäure, nach unsern bisherigen Erfahrungen keine andern Aminosäuren, insbesondere nicht Asparaginsäure und auch nicht Brenztraubensäure. Es eignet sich deshalb zur Bestimmung von L-Glutaminsäure in Zellextrakten. Indem wir aus Zellextrakten mit Trockenpulver Kohlensäure entwickelten und mit Hilfe dieser Tests die Substanz isolierten, aus denen die Kohlensäure stammte, fanden wir, daß die Quelle der Kohlensäure Glutaminsäure ist. - Die Suspension des Trockenpulvers in Wasser wird durch $10^{-3}-n$. Blausäure inaktiviert, durch Erwärmen auf $70^{\circ}$ schnell unwirksam.

Zur Bestimmung der Glutaminsäure brachten wir $20 \mathrm{mg}$ Trockenpulver in die Birne eines kegelförmi- gen Manometriegefäßes. Der Hauptraum enthielt $3 \mathrm{~cm}^{3}$ der zu bestimmenden Lösung. $p_{\mathrm{H}}$ nach Zugabe des Trockenpulvers war 5,0, die Temperatur war $20^{\circ}$, der Gasraum enthielt Argon. Abb. 1 zeigt das Ergebnis eines Versuchs, bei dem die zugesetzten $4,8 \mu$ Mole Glutaminsäure nach 50 Min. zur Hälfte zersetzt worden waren.

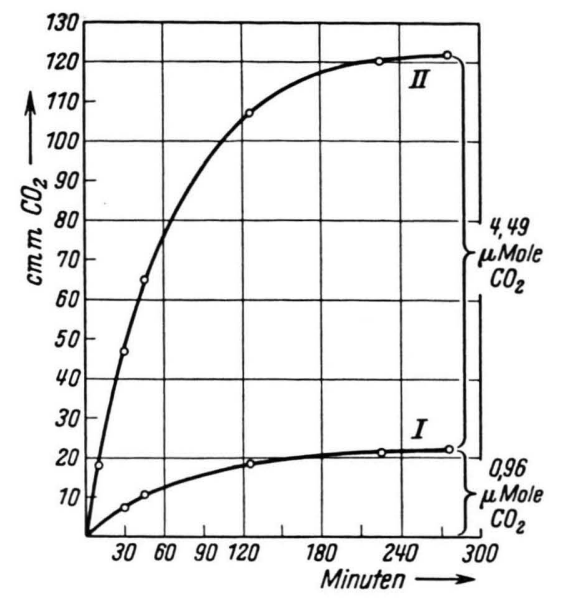

Abb. 1. Decarboxylierung der L-Glutaminsäure durch $20 \mathrm{mg}$ Chlorella-Trockenpulver. $20^{\circ}$. Anaerob. $p_{\mathrm{H}} 3,8 . \mathrm{I}: 3 \mathrm{~cm}^{3}$ Salzlösung S. II: $3 \mathrm{~cm}^{3}$ Salzlösung S+4,8 $\mu$ Mole L-Glutaminsäure.

Einer Erläuterung bedarf, warum ohne Zusatz von Glutaminsäure Kohlensäure entwickelt wird. Diese Kohlensäure stammt aus der Glutaminsäure des Trockenpulvers. Die Glutaminsäure ist in dem Trockenpulver fest gebunden und kann durch Wasser, sogar bei $100^{\circ}$, nur schwer extrahiert werden. Die so bestimmte Glutaminsäure betrug in $20 \mathrm{mg}$ Trockenpulver 1 bis 1,5 $\mu$ Mole.

Beim Erhitzen frischer Zellen jedoch, die in Wasser oder Salzlösung suspendiert sind, wird die Glutaminsäure extrahiert und kann in solchen Extrakten mit Chlorella-Trockenpulver bestimmt werden. Gibt man vor dem Erhitzen Fluorid zu den Zellen, wäscht dann das Fluorid wieder fort und erhitzt dann die Zellen, so kann man zeigen, daß die Glutaminsäure durch das Fluorid zersetzt worden ist.

Zum Beispiel wurden $500 \mathrm{~mm}^{3}$ Chlorella in $3 \mathrm{~cm}^{3}$ Salzlösung $\mathrm{S}^{*}$ suspendiert und $10 \mathrm{Min}$. auf $90^{\circ} \mathrm{er}$ hitzt. Dann wurde zentrifugiert und im überstehenden Hitzeextrakt die Glutaminsäure mit 20 mg Trokkenpulver, wie im Versuch der Abb. 1, bestimmt. Wir fanden bei $20^{\circ}$ in 275 Min. die folgenden Endwerte an Kohlensäure: 
aus reiner

Salzlösung

aus Hitzeextrakt der mit $n / 80-\mathrm{F}$ 15 Min.

vorbehandelt war

$+28 \mathrm{~mm}^{3} \mathrm{CO}_{2}$

$$
\begin{array}{ll}
+210 \mathrm{~mm}^{3} \mathrm{CO}_{2} & +75 \mathrm{~mm}^{3} \mathrm{CO}_{2} \\
-28 \mathrm{~mm}^{3} \mathrm{CO}_{2} & -28 \mathrm{~mm}^{3} \mathrm{CO}_{2} \\
\hline+182 \mathrm{~mm}^{3} \mathrm{CO}_{2} & +47 \mathrm{~mm}^{3} \mathrm{CO}_{2}
\end{array}
$$

Aus den $500 \mathrm{~mm}^{3}$ Chlorella waren also $7,3 \mu$ Mole Glutaminsäure beim Erhitzen extrahiert worden, das ist

$\frac{7,3 \cdot 0,147}{500 \cdot 0,225} 100=0,94 \%$ des Zell-Trockengewichts .

$74 \%$ davon waren durch das Fluorid zersetzt worden.

\section{Glutaminsäure, chromatographisch}

Um die Glutaminsäure, ihren Zerfall und Wiederaufbau, chromatographisch zu bestimmen, wurde zu $100 \mathrm{~mm}^{3}$ Chlorella, suspendiert in $3 \mathrm{~cm}^{3}$ Salzlösung $\mathrm{S}$, anaerob oder aerob Fluorid verschiedener Konzentration gegeben. $p_{\mathrm{H}}$ war immer 3,8 , die Temperatur immer $20^{\circ}$. War die Glutaminsäure anaerob zersetzt oder hatte sich aerob der stationäre Zustand zwischen Zerfall und Wiederaufbau eingestellt, so wurden die Zellen abzentrifugiert, mit $3 \mathrm{~cm}^{3}$ Wasser aufgewirbelt und 5 Min. auf $90^{\circ}$ erwärmt. Dann wurde wieder zentrifugiert, die überstehende Flüssigkeit im Vakuum eingetrocknet, ein Teil, der

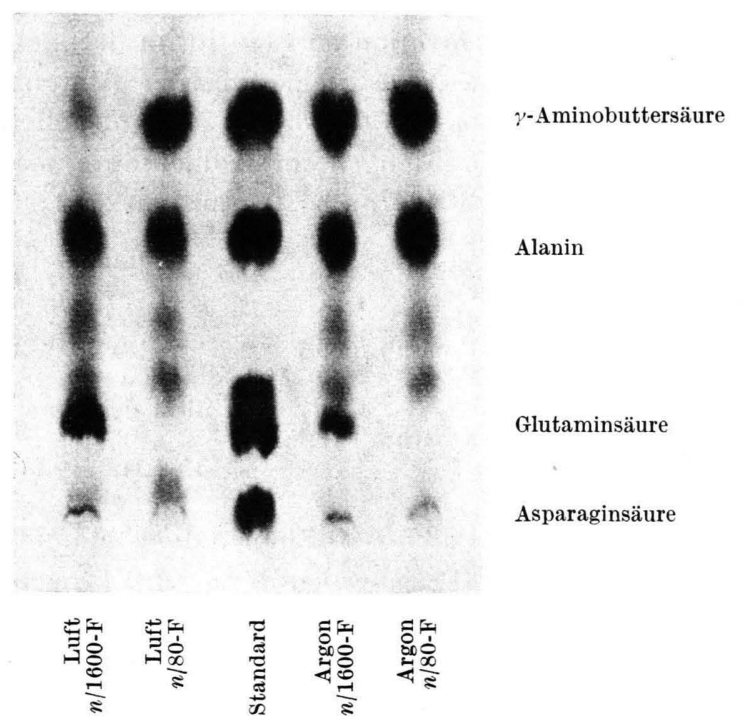

Abb. 2. Spaltung der Glutaminsäure in $\gamma$-Aminobuttersäure $+\mathrm{CO}_{2}$ anaerob durch $n / 1600$ - und $n / 80$-Fluorid. Aerob durch $n / 80$-Fluorid, aber nicht durch $n / 1600$-Fluorid.
$5 \mathrm{~mm}^{3}$ Zellen entsprach, auf Papier aufgetragen, mit Phenol-Citrat-Phosphat entwickelt und mit Ninhydrin besprüht. Die Abb. 2, 3 und 4 zeigen einige Ergebnisse. In der Standardspalte sieht man in aufsteigender Folge Asparaginsäure, Glutaminsäure, Alanin und $\gamma$-Aminobuttersäure.

Man beachte, daß man in keiner Abbildung eine deutliche Änderung der Konzentrationen der Asparaginsäure oder des Alanins sieht.

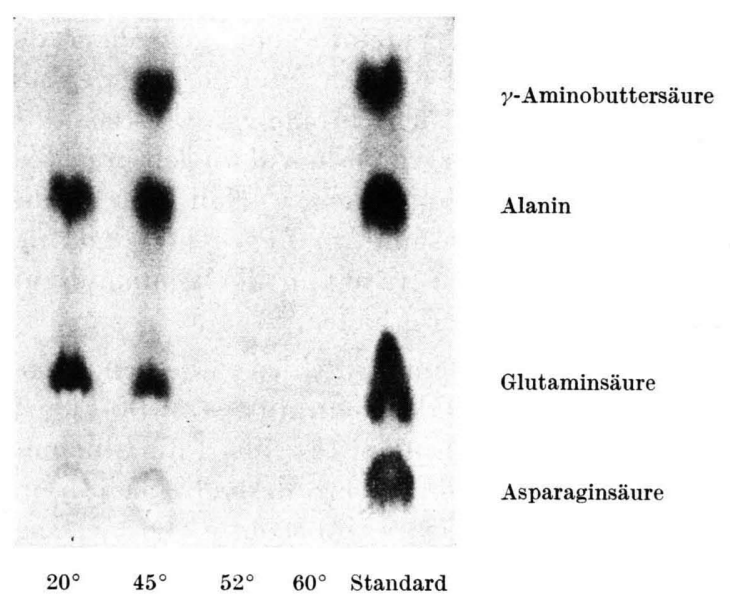

Abb. 3. Spaltung der Glutaminsäure in $\gamma$-Aminobuttersäure $+\mathrm{CO}_{2}$ durch Erwärmen auf $45^{\circ}$. Bei $52^{\circ}$ und $60^{\circ}$ ist nichts zu sehen, weil die Aminosäuren bereits durch die Salzlösung extrahiert worden sind.

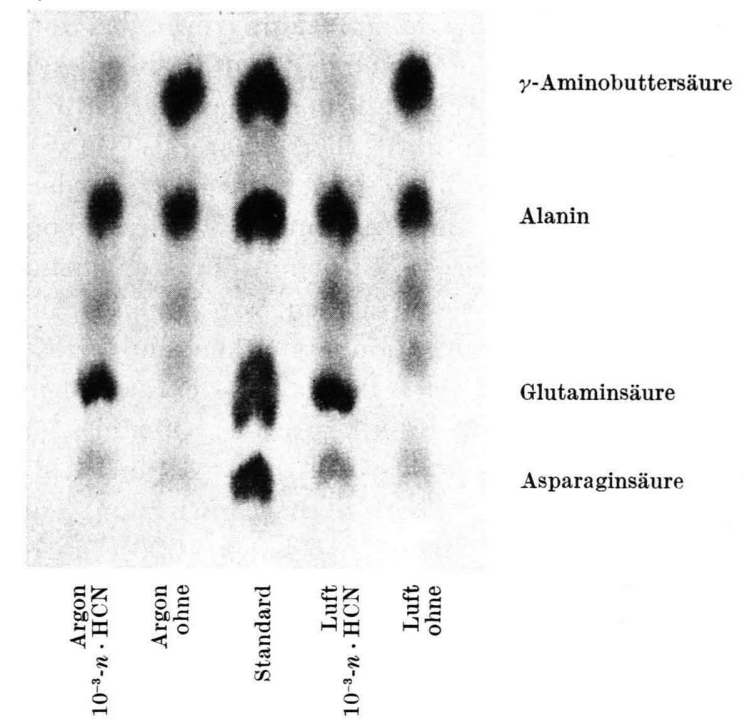

Abb. 4. n/80-Fluroid in Argon oder Luft \pm Blausäure. Die große Fluoridkonzentration $n / 80$ zersetzt in Argon wie in Luft alle Glutaminsäure. $10^{-3}$-Blausäure verhindert die Zersetzung anaerob wie aerob. 


\section{Glutaminsäure, manometrisch}

Auf Grund der beschriebenen Versuche ist es unzweifelhaft, daß die labile Kohlensäure ${ }^{2}$ der Chlorella, die sich bei Zusatz von Fluorid oder durch andere Maßnahmen entwickelt, aus der $\alpha$-Decarboxylierung der Glutaminsäure stammt, so daß man die Glutaminsäure in Chlorella manometrisch bestimmen kann, indem man Fluorid zu lebender Chlorella gibt und die Entwicklung der Kohlensäure mißt. Dies ist eine große Vereinfachung gegenüber den Versuchen mit Zellextrakten und Trockenferment und auch gegenüber den chromatographischen Versuchen. Durch Zusatz von Fluorid zu lebenden Zellen haben wir die Möglichkeit, Zerfall und Wiederaufbau der Glutaminsäure im Leben hervorzurufen und dadurch über die Funktion der Glutaminsäure im Leben Auskunft zu erhalten.

Man muß dabei unterscheiden zwischen hohen und niedrigen Fluoridkonzentrationen. $n / 80$-Fluorid ist eine hohe Konzentration, es zersetzt die Glutaminsäure irrerversibel und zersetzt deshalb anaerob und aerob die Glutaminsäure vollständig. Ordnet man dabei die Manometrie so an, daß $v_{\mathrm{F}}$ klein gegen $v_{\mathrm{G}}$ ist, so stört die Atmung in der kurzen Zeit der Glutaminsäurezersetzung nicht und man erhält dieselben Drucke an entwickelter Kohlensäure, ob der Gasraum Argon oder Luft enthält.

Kleinere Fluoridkonzentrationen, $n / 1000$ bis $n / 300$, zersetzen die Glutaminsäure reversibel und steigern außerdem die Atmung. Wenn die Bedingungen aerob sind, wird deshalb der Druck der entwik. kelten Kohlensäure in nicht übersehbarer Weise vermindert, so daß aerob die Glutaminsäure aus dem entwickelten Druck nicht bestimmt werden kann. Um trotzdem die aerobe Glutaminsäure-Zersetzung manometrisch zu messen, lassen wir zunächst die kleine Fluorid-Konzentration einwirken und zersetzen dann den Rest der Glutaminsäure durch viel Fluorid. Wenn z. B. $n / 80$-Fluorid aus $100 \mathrm{~mm}^{3}$ Zellen, die mit Luft gesättigt sind, direkt $40 \mathrm{~cm}^{3} \mathrm{Koh}$ lensäure entwickelt, aber nach Einwirkung von $n / 1000$-Fluorid nur $30 \mathrm{~mm}^{3}$, so hat $n / 1000$-Fluorid $1 / 4$ der Glutaminsäure zersetzt. Man beachte, daß bei dieser Anordnung alles aerob geschieht und somit alle Einwände, die mit der anaeroben Milchsäurebildung zusammenhängen, fortfallen.

Bei der ,aeroben“ Methode ist bei Sättigung mit Luft darauf zu achten, daß nach Schließen der Hähne nicht zu lange mit der Zugabe des Fluorids gewartet

wird. Andernfalls könnte es sein, daß infolge der Atmung der Kohlensäuredruck soweit steigt, daß sich merkliche Mengen an dissoziierender Kohlensäure in der Chlorella bilden (vgl. Abschnitt 6) .

\section{Zersetzung und Wiederaufbau der Glutaminsäure}

Während die Glutaminsäure anaerob durch sehr kleine Konzentrationen an Fluorid vollständig zersetzt wird, findet aerob eine Rückbildung von Glutaminsäure statt:

Glutaminsäure $\rightleftarrows \gamma$-Aminobuttersäure $+\mathrm{CO}_{2}$.

Indessen ist die Reaktion von rechts nach links keine einfache Rückreaktion, da die Reaktion anaerob vollständig in der Richtung der Zersetzung verläuft. Zur Rückreaktion, zum Wiederaufbau der Glutaminsäure, ist Sauerstoff, d. h. ist Atmung nötig und tatsächlich steigt die Atmung, wenn man aerob kleine Mengen Fluorid zu Chlorella hinzufügt. Zum Beispiel verbrauchten in $5 \mathrm{Min} .100 \mathrm{~mm}^{3}$ Chlorella, suspendiert in $3 \mathrm{~cm}^{3}$ Salzlösung S, bei $20^{\circ}$ in Luft die folgenden Mengen an Sauerstoff, wenn sich im Einsatz der Manometriegefäße Kalilauge befand:

$\begin{array}{lllc} & & \begin{array}{c}2 \text {-tägige Südzellen } \\ {\left[\mathrm{mm}^{3}\right]}\end{array} & \begin{array}{c}1 \text {-tägige A-Zellen } \\ {\left[\mathrm{mm}^{3}\right]}\end{array} \\ \text { ohne F } & 10^{\prime} & -10 & -5,2 \\ \text { in } n / 640-\mathrm{F} & 10^{\prime} & -12,5 & -9,4 \\ \downarrow & 10^{\prime} & -18,4 & -11,4 \\ \downarrow & 10^{\prime} & -17,9 & -12,7\end{array}$

Die gleiche Konzentration an Fluorid, in der gleichen Salzlösung, die diese Steigerung der Atmung bewirkte, entwickelte aus Chlorella anaerob oder aerob die folgenden Mengen an Kohlensäure aus Glutaminsäure (Endwerte nach $20 \mathrm{Min}$. bei $20^{\circ}$ ) :

$\begin{array}{ll}\text { anaerob durch } & \left\{\begin{array}{l}n / 72-\mathrm{F} \\ n / 640-\mathrm{F} \\ n / 72-\mathrm{F} \text { nach } n / 640-\mathrm{F}\end{array}\right. \\ \text { aerob durch } & \left\{\begin{array}{l}n / 72-\mathrm{F} \\ n / 640-\mathrm{F} \\ n / 72-\mathrm{F} \text { nach } n / 640-\mathrm{F}\end{array}\right.\end{array}$

also aerob durch ' $n / 640-\mathrm{F}$

$\left[\mathrm{mm}^{3}\right]$
42
42
0
42
nicht gemessen
32,9
$42-31,9=9,1$

Das Ergebnis ist, daß $n / 640$ Fluorid die Glutaminsäure anaerob zu $100 \%$, aber aerob nur zu $21 \%$ zersetzt. Aus dem so gefundenen stationären Zustand zwischen Zersetzung und Wiederaufbau der Glutaminsäure und aus der Geschwindigkeit der anaeroben Zersetzung der Glutaminsäure kann man berechnen, $\mathrm{da} ß$ aerob (in $n / 640$-Fluorid) pro Stunde das $2^{1 / 2}$ fache Zellvolumen an Kohlensäure fixiert wird. 


\section{Dissoziierende Kohlensäure}

Sättigt man Chlorella mit verschiedenen Kohlensäuredrucken und gibt dann Fluorid hinzu, so wird nicht nur Kohlensäure aus der Glutaminsäure entwickelt, sondern auch aus Bicarbonat, das durch Umsetzung mit Salzen schwacher Säuren entstanden ist, vielleicht auch aus Carbaminsäuren, die durch die S i e g f ri e d sche Reaktion in Chlorella entstanden sind. Diese Kohlensäure ist abhängig vom Kohlensäuredruck, während die aus Glutaminsäure entwickelte Kohlensäure unabhängig vom Kohlensäuredruck ist.

Bei diesen Versuchen haben wir die bisher chemisch noch nicht erklärte Tatsache gefunden, daß aerob viel mehr Kohlensäure dissoziierend gebunden wird als anaerob. Wurden z. B. $100 \mathrm{~mm}^{3}$ 2-tägige Südzellen, die $1,84 \mu$ Mole Chlorophyll enthielten und in $3 \mathrm{~cm}^{3}$ Salzlösung $\left(p_{\mathrm{H}} 3,8\right)$ suspendiert waren, mit Kohlensäuredrucken von $1 / 20$ bis $1 / 2$ Atmosphäre gesättigt, so trieb $n / 80$-Fluorid bei $20^{\circ}$ in 20 Min. die folgenden Mengen an Kohlensäure aus (Endwerte) :

$\begin{array}{cccccc}\mathrm{CO}_{2} \text {-Druck [Atm] } & 0 & 1 / 20 & 1 / 10 & 1 / 5 & 1 / 2 \\ \begin{array}{c}\text { Anaerob } \mathrm{CO}_{2}\left[\mathrm{~mm}^{3}\right] \\ \left(\mathrm{CO}_{2} \text {-Argon }\right)\end{array} & 38,8 & 44,7 & 48,2 & 51,8 & 56,3 \\ \begin{array}{c}\text { Aerob } \mathrm{CO}_{2}\left[\mathrm{~mm}^{3}\right] \\ \left(25 \text { Vol.-Proz. } \mathrm{O}_{2}\right)\end{array} & 38,0 & 51,5 & 63,0 & 79,5 & 97\end{array}$

Zieht man hier von den anaeroben Werten die aus Glutaminsäure beim $\mathrm{CO}_{2}$-Druck Null entwickelte Kohlensäure $a b$, so erhält man die ,anaerobe dissoziierende $\mathrm{CO}_{2}{ }$; und zieht man von den aeroben Werten die anaeroben Werte $a b$, so erhält man diejenige $\mathrm{CO}_{2}$, die nur unter dem Einfluß des Sauerstoffs gebunden wird und die wir die nur „aerob dissoziierende $\mathrm{CO}_{2}$ " nennen wollen. Die beiden Subtraktionen ergeben:

$\begin{array}{lrrlc}\mathrm{CO}_{2} \text {-Druck }[\text { Atm }] & 1 / 20 & 1 / 10 & 1 / 5 & 1 / 2 \\ \text { Anaerob } \mathrm{CO}_{2}\left[\mathrm{~mm}^{3}\right] & 5,9 & 9,4 & 13 & 17,5 \\ \text { Aerob } \mathrm{CO}_{2}\left[\mathrm{~mm}^{3}\right] & 6,8 & 14,8 & 27,7 & 40,7\end{array}$

Das Ergebnis ist in Abb. 5 graphisch dargestellt. Man sieht, daß anaerob wie aerob der Sättigungswert bei einem $\mathrm{CO}_{2}$-Druck von einer halben Atm erreicht wird; er ist für die nur aerob dissoziierende $\mathrm{CO}_{2} 2^{1 / 2}$-mal so groß, wie für die anaerobe dissoziierende $\mathrm{CO}_{2}$.

Vergleicht man die Dissoziationskurven der nur aerob dissoziierenden $\mathrm{CO}_{2}$ für verschieden gezüchtete Chlorella, so findet man große Unterschiede. 1-tägige A-Zellen - die die besten Quantenausbeuten

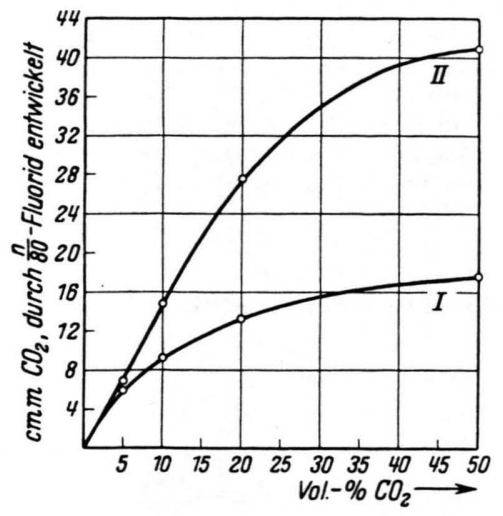

Abb. 5. Disoziierende $\mathrm{CO}_{2}$, durch $n / 80$-Fluorid bei $p_{\mathrm{H}} 3,8$ und $20^{\circ}$ aus Chlorella entwickelt. I: anaerob dissoziierende $\mathrm{CO}_{2}$. II: nur aerob dissoziierende $\mathrm{CO}_{2}$.

geben - binden die aerobe $\mathrm{CO}_{2}$ fester, als 2-tägige Südzellen, die eine schlechtere Quantenausbeute geben. Zum Beispiel fanden wir die folgenden Sättigungsgrade der nur aerob dissozierenden $\mathrm{CO}_{2}$ :

$\begin{array}{lcccc}\mathrm{CO}_{2} \text { Druck [Atm] } & 1 / 20 & 1 / 10 & 1 / 5 & 1 / 2 \\ \begin{array}{c}\text { \% Sättigung in } \\ \text { 1-täg. A-Zellen }\end{array} & 65 & 72 & 84 & 100 \\ \begin{array}{c}\text { \% Sättigung in } \\ \text { 2-täg. Südzellen }\end{array} & 17 & 36 & 68 & 100\end{array}$

Eine weitere interessante Eigenschaft der nur aerob dissozierenden Kohlensäure ist, daß der Sättigungswert gleich dem Glutaminsäuregehalt der Zellen ist. In dem Versuch der Abb. 5 betrug der Gehalt an Glutaminsäure $38,4 \mathrm{~mm}^{3}$, der Sättigungswert der nur aerob dissozierenden $\mathrm{CO}_{2} 41 \mathrm{~mm}^{3} \mathrm{CO}_{2}$, der Chlorophyllgehalt $41,2 \mathrm{~mm}^{3}$. Glutaminsäuregehalt, Chlorophyllgehalt und Sättigungswert der nur aerob dissozierenden $\mathrm{CO}_{2}$ waren also innerhalb der Fehlergrenzen gleich.

Dieselben 3 Größen bestimmten wir für $\mathrm{x}$-Zellen, die 5-mal soviel Glutaminsäure als Chlorophyll enthielten. Wir fanden in $100 \mathrm{~mm}^{3} \mathrm{x}$-Zellen:

Sättigungswert der nur aerob

dissoziierenden $\mathrm{CO}_{2}$ :

$[\mu \mathrm{Mole}]$

Glutaminsäure :

1,9

Chlorophyll:

0,418

In solchen Zellen also, in denen Chlorophyll- und Glutaminsäuregehalt verschieden sind, ist der Sättigungswert der nur aerob dissozierenden $\mathrm{CO}_{2}$ gleich dem Glutaminsäure- und nicht gleich Chlorophyllgehalt.

Anmerkung. Als wir den Einfluß des Sauerstoffs auf die dissoziierende $\mathrm{CO}_{2}$ fanden, dachten wir zunächst, daß 
der anaerobe Wert der disoziierenden $\mathrm{CO}_{2}$ kleiner gefunden wird, weil anaerob in der Sättigungszeit die gebildete Milchsäure bereits einen Teil der $\mathrm{CO}_{2}$ austreibt. Durch Versuche mit 2-tägigen Südzellen, die anaerob nur sehr langsam Milchsäure bilden und durch Variation der Sättigungszeiten konnte dieser Einwand ausgeschlossen werden.

\section{Glutaminsäure und Lichtwirkung}

Bereits in unsrer vorigen Mitt. ${ }^{2}$ haben wir beschrieben, wie man durch steigende Konzentrationen von Fluorid die Lichtwirkung in Chlorella in zunehmendem $\mathrm{Ma} \beta$ hemmen kann. Wir können dieses Ergebnis heute so ausdrücken, daß mit zunehmendem Zerfall der Glutaminsäure die Lichtwirkung mehr und mehr gehemmt wird. Den Zusammenhang zwischen Glutaminsäure und Lichtwirkung haben wir jetzt quantitativ untersucht, indem nach der Messung der Lichtwirkung der Rest an Glutaminsäure durch Zusatz von viel Fluorid gemessen wurde. So konnten wir für verschiedene (kleine) Konzentrationen an Fluorid Glutaminsäuregehalt und Lichtwirkung quantitativ vergleichen. Zum Beispiel fanden wir, wenn aerob mit $n / 640$ - und $n / 320$-Fluorid der stationäre Zustand zwischen Zerfall und Wiederaufbau der Glutaminsäure hergestellt worden war:

$\begin{array}{ccc}\begin{array}{c}\text { Konzentration } \\ \text { des Fluorids }\end{array} & \begin{array}{c}\text { Glutaminsäure- } \\ \text { Rest }\end{array} & \begin{array}{c}\text { Rest der } \\ \text { Lichtwirkung }\end{array} \\ & {[\%]} & {[\%]} \\ n / 640 & 79 & 82 \\ n / 320 & 36 & 36\end{array}$

wobei die Lichtwirkung, in $10 \mathrm{Vol}$.Proz. $\mathrm{CO}_{2}$ 30 Vol-.Proz. $\mathrm{O}_{2}$, mit den Intensitäten $J=430$ oder $J=57 \mathrm{~mm}^{3}$ Quanten pro Minute bestimmt wurde.

Den Einwand, das Fluorid hemme die Lichtwirkung nicht wegen der Zersetzung der Glutaminsäure, sondern aus andern Gründen, kann man ausschließen, indem man die Hemmung der Lichtwirkung durch ein und dieselbe Fluoridkonzentration anaerob und aerob vergleicht, z. B. durch $n / 1000$ Fluorid, das anaerob alle Glutaminsäure, aber aerob nur wenig Glutaminsäure zersetzt. Dann findet man, - bei der gleichen Fluoridkonzentration - anaerob eine sehr große und aerob eine sehr kleine Hemmung der Lichtwirkung.

Es lag daraufhin nahe zu prüfen, ob die Glutaminsäure beim Belichten von Chlorella abnimmt. Wir haben in keinem Fall eine Abnahme gefunden in Versuchen, in denen die Glutaminsäure nach langer
Verdunkelung oder nach Belichtung sowohl manometrisch mit Fluorid als auch chromatographisch bestimmt wurde.

\section{Dissoziierende Kohlensäure und Lichtwirkung}

Während die Glutaminsäure im Licht nicht abnimmt, fanden wir, daß die aerobe dissoziierende Kohlensäure im Licht sehr stark abnimmt. Die Versuche wurden mit 1-tägigen A-Zellen ausgeführt, von denen $100 \mathrm{~mm}^{3}$ in $3 \mathrm{~cm}^{3}$ Salzlösung $\mathrm{S}\left(p_{\mathrm{H}} 3,8\right)$ suspendiert waren und die mit 20 oder 30 Vol.-Proz. $\mathrm{CO}_{2} 25$ Vol.-Proz. $\mathrm{O}_{2}$ gesättigt waren. Zum Beispiel entwickelte $n / 80$-Fluorid die folgenden Mengen an Kohlensäure:

$$
\text { aus verdunkelten Zellen } \begin{gathered}
\text { aus Zellen, die } 2 \mathrm{Min} \text {. mit } \\
J=30 \mathrm{~mm}^{3} \text { Quanten/Min. } \\
\text { belichtet worden waren }
\end{gathered}
$$

oder, wenn mit sehr hoher Intensität belichtet wurde

$$
\begin{array}{ccc}
\text { aus verdunkelten } & \text { nach } 1 \text { Min. } & \text { nach } 5 \mathrm{Min} \\
\text { Zellen } & J=800 & J=800 \\
77 \mathrm{~mm}^{3} \mathrm{CO}_{2} & 62 \mathrm{~mm}^{3} \mathrm{CO}_{2} & 49 \mathrm{~mm}^{3} \mathrm{CO}_{2}
\end{array}
$$

Die dissoziierende Kohlensäure nahm also bei Belichtung sehr erheblich ab. Wurden die im 2. Versuch verwendeten Zellen, nachdem sie 5 Min. mit der hohen Intensität $J=800$ Quanten/Min. belichtet worden waren, verdunkelt und wurde dann nach verschiedenen Dunkelzeiten das Fluorid zu den Zellen gegeben, so entwickelte $n / 80$-Fluorid nach wachsenden Dunkelzeiten die folgenden Mengen an Kohlensäure:

$\begin{array}{lcccccc}\text { Min.: } & 0 & 1 & 5 & 10 & 20 & \text { dunkel } \\ \mathrm{CO}_{2}\left[\mathrm{~mm}^{3}\right]: & 49 & 46 & 62 & 72 & 75 & \end{array}$

Der Hellwert an dissoziierender $\mathrm{CO}_{2}$ stieg also im Lauf von 20 Dunkelminuten wieder auf den ursprünglichen Dunkelwert.

Zusammengefaßt ist das Ergebnis der Versuche: Entwickelt man durch Belichtung vorher verdunkelter Zellen die Sauerstoffkapazität ${ }^{9}$ der Chlorella, so nimmt während der stöchiometrischen Sauerstoffentwicklung die dissoziierende Kohlensäure sehr stark ab. Im Dunkeln, in der Zeit, in der die induzierte Atmung die Sauerstoffkapazität wieder herstellt, steigt die dissoziierende Kohlensäure wieder auf ihren Anfangswert. 